\title{
A fiber-integrated optical component fabricated via photopolymerization: Mode-selective grating coupler
}

\author{
Can Sümer ${ }^{\mathrm{a}, \mathrm{b}, *}$, M. Salih Dinleyici ${ }^{\mathrm{a}}$ \\ ${ }^{a}$ Electrical and Electronics Engineering, Izmir Institute of Technology, Gülbahçe-Urla, 35430 Izmir, Turkey \\ ${ }^{\mathrm{b}}$ Lead Technologies Group, Vestel Electronics, Vestel City, 45030 Manisa, Turkey
}

\section{A R T I C L E I N F O}

\section{Article history:}

Received 19 May 2013

Received in revised form

12 June 2013

Accepted 14 June 2013

Available online 4 July 2013

Keywords:

Photopolymer

Direct-writing

Surface relief

Grating coupler

Mode-selective coupling

\begin{abstract}
A B S T R A C T
We demonstrate a mode-selective directional coupler based on a grating structure, which is fabricated by laser direct-writing on a photopolymer thin film. The device is implemented on the flat planar surface of the D-Fiber, enabling fiber integration, where an Acrylamide/Polyvinyl Alcohol based photopolymer material is used in the fabrication of the device. While the refractive index modulation properties of the polymer material are well known, surface relief and corrugation properties due to photopolymerization are investigated in this study. Theoretical model of the device is presented together with the optimization and simulation results of the final device; experimental results have been found to be in good agreement with simulations.
\end{abstract}

(c) 2013 Elsevier B.V. All rights reserved.

\section{Introduction}

Photopolymerization is a popular research area and a practical tool for the fabrication of optical components; the focus is particularly common in creation of holographic memory systems $[1,2]$. The main reasons for the popularity are the low-cost material, simple handling and easy production. Apart from holographic techniques, direct-writing is an alternative method for fabrication of optical components, which provides more control and precision in production, and also allows for the fabrication of three-dimensional components [3].

Grating couplers are one of the key components of optical networks. Being a passive component allows for the fundamental nodes of many all-optical devices to be fabricated by utilizing grating couplers, including wavelength filters, all-optical switches, etc. [4-6]. D-Fiber is a special kind of optical fiber with an outer form of cladding layer resembling the letter $\mathrm{D}$, where the flat planar side surface of the fiber allows for the implementation of various structures on the fiber and facilitates fiber-integration of optical devices like sensors, etc. [7,8].

In this paper, we present a fiber-integrated mode-selective directional coupler mechanism which is based on a grating

\footnotetext{
* Corresponding author at: Vestel Electronics Corporation, Lead Technologies Group, Vestel City - Organize Sanayi Bol, 45030 Manisa, Turkey. Tel.: +90 236231 06 60; fax: +902362130548.

E-mail address: cansumer@iyte.edu.tr (C. Sümer).
}

structure. The device is fabricated via laser direct-writing of a photopolymer thin film fabricated on the flat planar surface of a D-Fiber. The theoretical model of the device has been adapted from the earlier work of Yariv and Yeh [9] and the design optimization of the device has been performed by utilizing the waveguide separation and the device length as optimization parameters.

The off-the-shelf polymer recipe and the refractive index change of the polymer material have been investigated elsewhere [10]; however, the investigation and mathematical modeling of the surface relief and corrugation properties due to photopolymerization process are performed in this study.

\section{Properties of photopolymer gratings}

The majority of photopolymer optical components stem from research aimed at obtaining memory units based on polymer materials, fabricated via holographic technique [11,12]. On the other hand, direct-writing method makes possible the fabrication of planar lightwave circuits on thin film polymer layers [10,13].

Volumetric refractive index modulation and surface modification effects of polymerization need to be taken into account during the design and fabrication of photopolymer optical devices [13]. Photopolymerization achieved through laser direct-writing exhibits a non-local behavior, in that the induced material modification is not limited to the area exposed to the laser beam [14]. The firstharmonic diffusion model demonstrated by Gallego et al. estimates 
the performance of holographic gratings fabricated in photopolymer materials with respect to the thickness of the polymer film layer [15]. Accordingly, the rate of polymerization increases with increased film thickness; so does the rate of monomer diffusion during photopolymerization. The two-way diffusion model introduced by Babeva et al. accounts for not only the diffusion mechanism taking place during the photopolymerization process, but also the post process stabilization of the material subsequent to laser direct-writing [16]. The study reveals an inverse correlation between the direct-writing beam intensity and the surface relief profile as a result of the photopolymerization.

An Acrylamide based Polyvinyl Alcohol (PVA) matrix photopolymer was used to prepare the photopolymerizable thin films used in this study. Films were prepared using the recipe that had been studied and finalized earlier, which was shown to achieve a refractive index difference of 0.0013 through laser direct-writing [10], where, the volumetric profile of refractive index modulation induced through the process was also demonstrated. The polymer material was prepared by adding $2.967 \% \mathrm{w} / \mathrm{v}$ Triethanolamine used as co-initiator, $3.170 \% \mathrm{w} / \mathrm{v}$ Acrylamide as the photo-active monomer and $0.016 \% \mathrm{w} / \mathrm{v}$ Yellowish Eosin used as photoinitiator into $10 \% \mathrm{w} / \mathrm{v}$ aqueous solution of PVA used as matrix. Upon mixing the materials, prepared solution was coated on flat side surfaces of optical fibers via direct casting, where the film thickness was adjusted subsequent to polymer casting via Doctor Blade method, using the apparatus allowing thickness adjustments in micrometer scale. Polymer coated fibers were left in a dark ambient for $24 \mathrm{~h}$ to allow for the PVA cure.

Grating structures were fabricated on a step-by-step basis, by direct-writing each grating step in an orderly fashion. Directwriting was performed using a $0.5 \mathrm{~mW} \mathrm{CW} \mathrm{He-Ne} \mathrm{laser} \mathrm{operating}$ at $543 \mathrm{~nm}$. Laser output was focused on the film surface via a $40 \times$ objective lens resulting in a $125 \mu \mathrm{m}$ spot-size, where the position and scan velocity of the focused beam was controlled using a computer-controlled xyz-stage (see Fig. 4.1).

The resultant grating-step surface profiles were investigated using a Stylus Surface Profilometer and the beam-velocity-dependent grating-step surface profiles were obtained. The resultant profiles, i.e., line shapes and peak corrugation amplitudes, were acquired with respect to surface-scanning speed of the direct-writing laser beam. The resultant grating-step surface profiles were curve-fitted using a piecewise Gaussian function comprising two separate curves, i.e., a narrower function for the middle region and a wider spread function for the tail regions (see Fig. 2.1), where both functions are of the form $G_{i}(x)=A_{i} \cdot \exp \left(-x^{2} / b_{i}^{2}\right), \quad i=1,2$; with $b_{i}{ }^{2}$ signifying the variance of the corresponding Gaussian function.

The dependence of the curve parameters of these piecewise Gaussian-type grating step surface profiles were obtained with respect

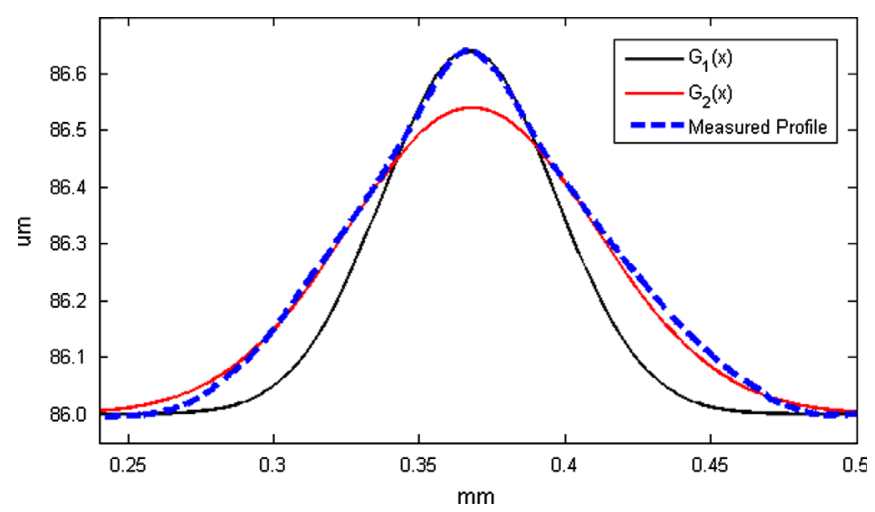

Fig. 2.1. Gaussian function fit for a single step of direct-written grating for beam speed $2.8 \mathrm{~mm} / \mathrm{s}$. to beam scan speed. Figs. 2.2 and 2.3 depict the direct-writing beam scan-speed dependence of the Gaussian fit coefficients and the grating step amplitude, respectively.

Based on the studies reported earlier [10], whole region of grating fabrication is refractive-index-modulated with a refractive index difference of 0.0013 . As presented in Fig. 2.3, the surface profile analysis of the fabricated waveguides, i.e., single lines, reveal an offset surface property of photopolymer structures. Accordingly, in the gratings (which are defined as repetitive parallel waveguides), while the corrugations caused by the surface relief follow the grating period, the corrugations themselves additionally exhibit an inherent height offset. Therefore, once the first grating step is written on the photopolymer film at the beginning of the grating, the following step right after the first step is then written on the surface which is already altered due to the writing of the previous grating step. The result is the cumulative height offset of the whole grating. This property was verified by surface profile measurements covering both the corrugations of the grating and the beginning of the grating region on the sample surface; where, a stylus profiler with a horizontal step size of $0.5 \mu \mathrm{m}$ was used in the measurements (see Fig. 2.4).

According to the results, the cumulative offset value of the surface relief depends on the velocity of the direct-writing laser beam. In addition, comparing the measurements for the offset with the previous measurements for the individual steps and waveguides shows that offset behavior measured above has been found to be in strong agreement with previous surface profile measurements.

The characteristics given in the above figures, together with the refractive index modulation reported earlier [10] provide the full set of fabrication abilities experimentally achievable using the given polymer recipe and the experimental means.

\section{Theoretical model}

\subsection{Length-dependent field amplitudes}

The schematic representation of the grating coupler is presented in Fig. 3.1; the device consists of a photopolymer periodic grating structure placed between two circular-core D-Fibers. The general concept of mode-selective coupling is that when two modes, the fundamental $\mathrm{LP}_{01}$ mode and the second $\mathrm{LP}_{11}$ mode, are excited in the input fiber, the coupler should couple the second mode into the output fiber (Output B) with maximum efficiency while the fundamental mode remains unperturbed, exiting the device through Output A.

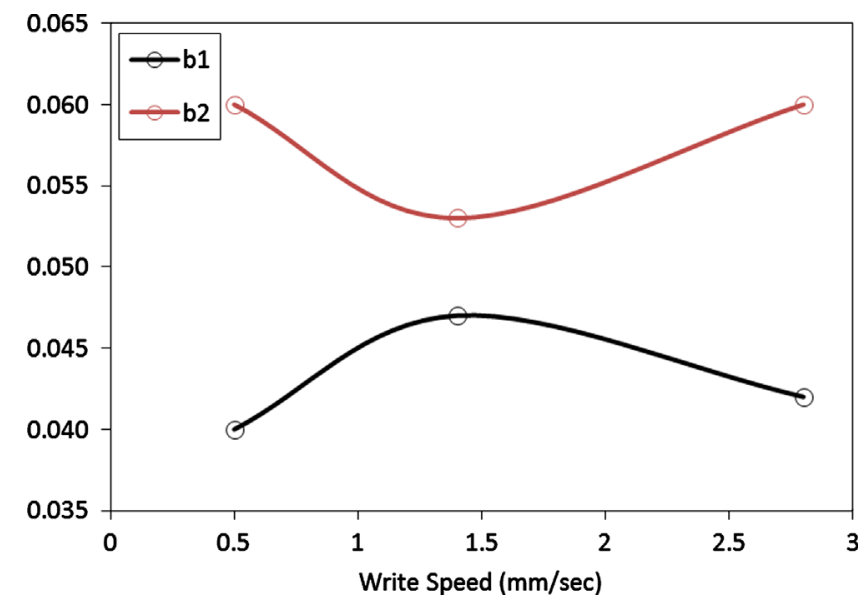

Fig. 2.2. Gaussian fit coefficients vs. direct-writing beam speed. 


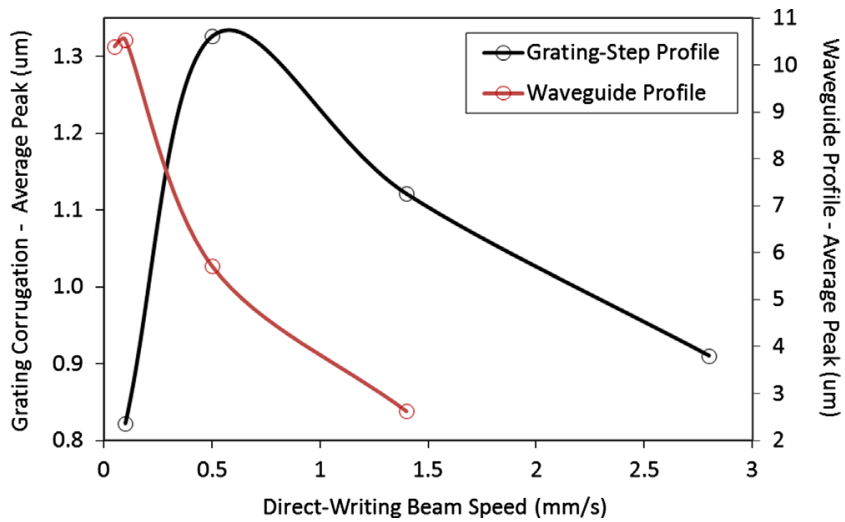

Fig. 2.3. Amplitude of grating steps/single lines vs. direct-writing beam speed.

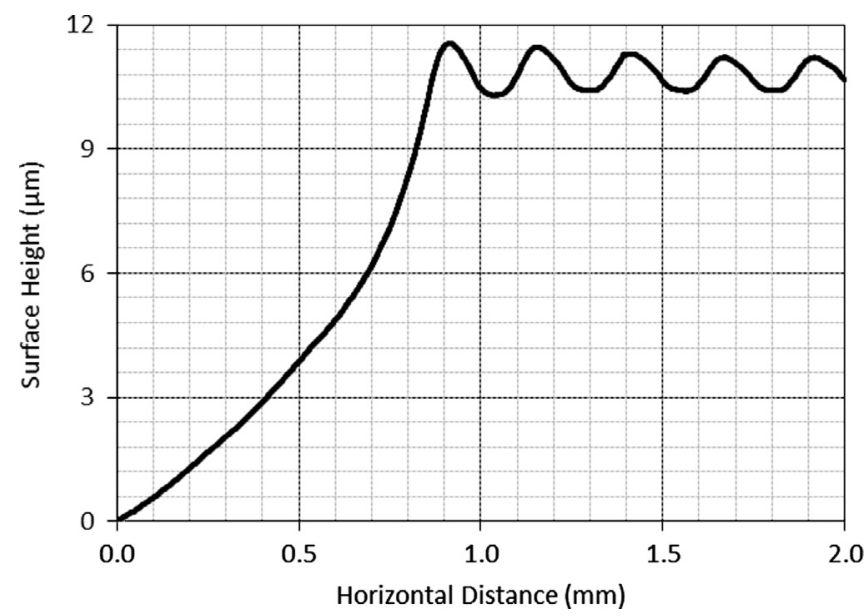

Fig. 2.4. Grating surface profile for direct-writing beam speed $0.1 \mathrm{~mm} / \mathrm{s}$.

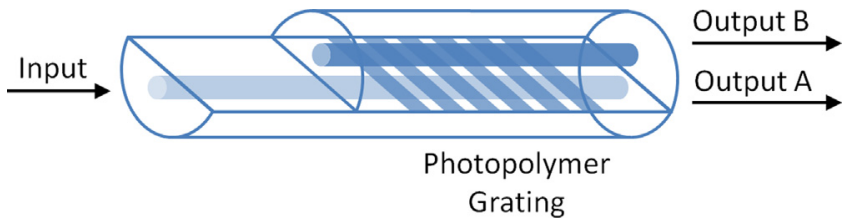

Fig. 3.1. Photopolymer mode-selective grating coupler.

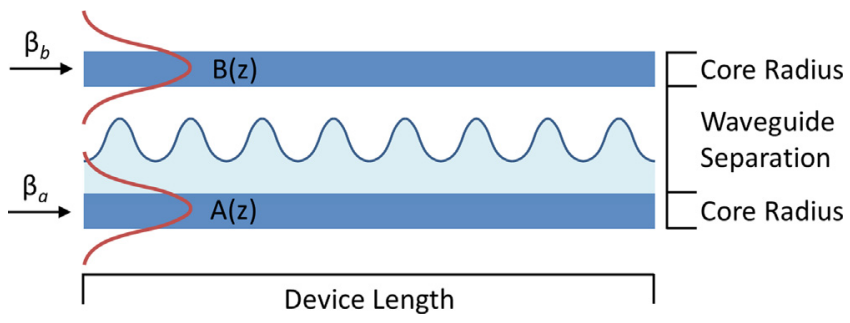

Fig. 3.2. Parameters regarding the theoretical model of the coupler.

The theoretical analysis and design of the grating coupler is based on the studies of Yariv and Yeh, [9] where the propagation of coupled waves in periodic structures is investigated. Adapting the model to the device, when the total electric field of the propagating modes is given as:

$\boldsymbol{E}(x, y, z)=A(z) \boldsymbol{\varepsilon}_{\boldsymbol{a}}(x, y) e^{-i \beta_{a} z}+B(z) \boldsymbol{\varepsilon}_{\boldsymbol{b}}(x, y) e^{-i \beta_{b} z}$

The length-dependent solutions of the amplitudes in the presence of the periodic photopolymer grating structure between the fibers are obtained as:

$$
\begin{aligned}
& A(z)=e^{i(\Delta \beta / 2) z}\left[\left(\cos s z-i \frac{\Delta \beta}{2} \frac{\sin s z}{s}\right) A(0)\right] \\
& B(z)=e^{-i(\Delta \beta / 2) z}\left[-i \kappa^{*} \frac{\sin s z}{s} A(0)\right]
\end{aligned}
$$

where,

$s^{2}=\kappa^{*} \kappa+\left(\frac{\Delta \beta}{2}\right)^{2}$

$\boldsymbol{\kappa}=\frac{\omega}{4} \int \boldsymbol{\varepsilon}_{\boldsymbol{a}}^{*}(x, y) \epsilon_{m}(x, y) \boldsymbol{\varepsilon}_{\boldsymbol{b}}(x, y)$

$\Delta \beta=\beta_{a}-\beta_{b}-m \frac{2 \pi}{\Lambda}=0$

in the above equations, $\boldsymbol{\kappa}$ is the coupling coefficient expressed through the overlap integral with $\epsilon_{m}(x, y)$ signifying the $m$ th Fourier component of the periodic grating structure. The Fourier components of the grating structure were obtained using the standard Fourier series expansion:

$F_{g}(z)=\frac{a_{0}}{2}+\sum_{n=1}^{N}\left[a_{n} \cos (n z)+b_{n} \sin (n z)\right]$

where,

$a_{n}=\frac{1}{L} \int_{-L}^{L} g(z) \cos (n z) d z, n \geq 0$

$b_{n}=\frac{1}{L} \int_{-L}^{L} g(z) \sin (\mathrm{nz}) d z, n \geq 1$

are the Fourier coefficients obtained as given in Fig. 3.3.

As seen in the figure, the physical grating structure is predominantly composed of $\cos (z)$ and $\cos (2 z)$ components.

\subsection{Propagation constants and phase matching condition}

The theoretical model requires the modes to be coupled in the input and output waveguides to be phase-matched. Let $\lambda_{0}$ be the wavelength of light, $n_{a}$ and $n_{b}$ be the effective indices of modes; then the phase difference between the two modes is:

$\Delta \beta=\beta_{a}-\beta_{b}-m \frac{2 \pi}{\Lambda}=n_{a} \frac{2 \pi}{\lambda_{0}}-n_{b} \frac{2 \pi}{\lambda_{0}}-m \frac{2 \pi}{\Lambda}=0$

In order to obtain co-directional coupling, the grating period should satisfy:

$\Lambda=m \frac{\lambda_{0}}{\left|n_{a}-n_{b}\right|}, \quad m=1,2,3 \cdots$

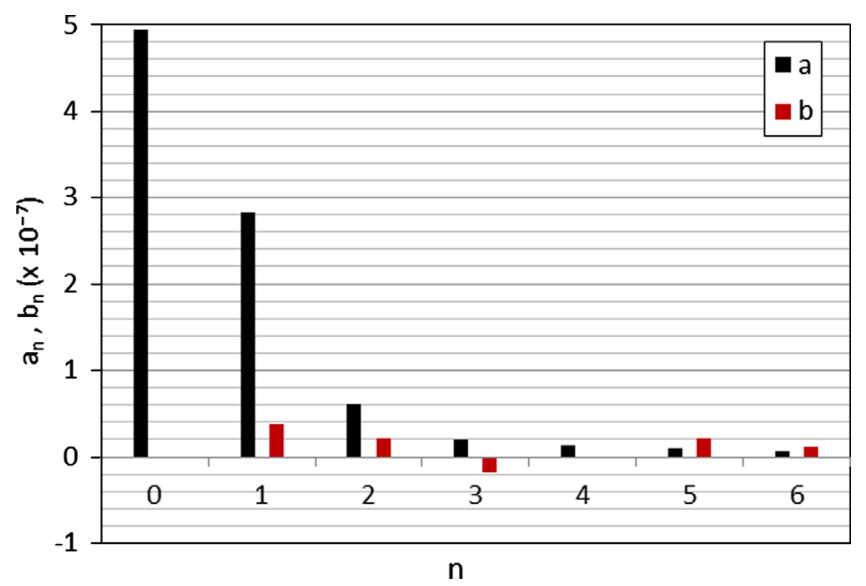

Fig. 3.3. $a_{n}$ and $b_{n}$ Fourier coefficients of the periodic grating structure. 
Optical fibers used in the design of the coupler have core refractive index $n_{\text {Core }}=1.4572$, cladding refractive index: $n_{\text {Clad }}=1.4507$ and core radius: $a=2.65 \mu \mathrm{m}$. When excited with a $632.8 \mathrm{~nm}$ laser, the fiber supports two modes, i.e., $\mathrm{LP}_{01}$ mode and $\mathrm{LP}_{11}$ mode, where the

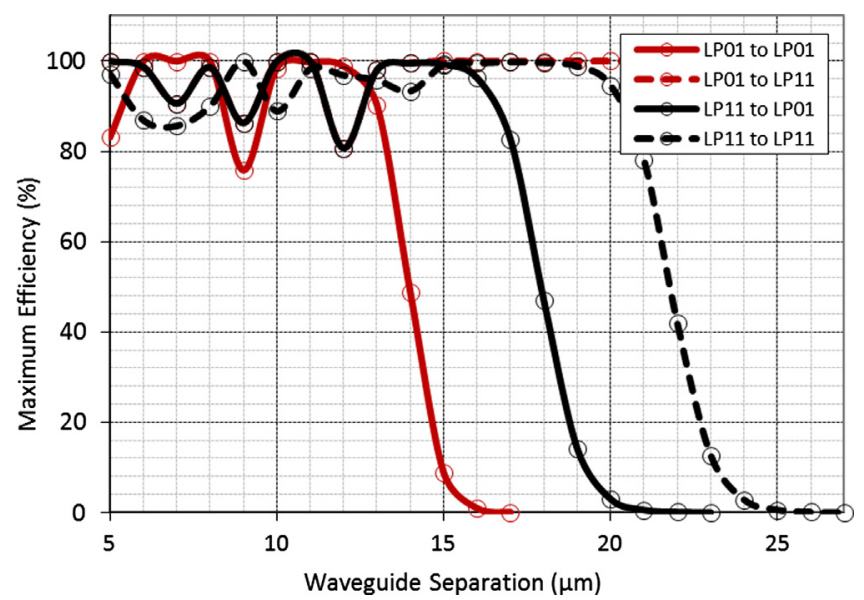

Fig. 3.4. Maximum achievable coupling efficiency with respect to waveguide separation.

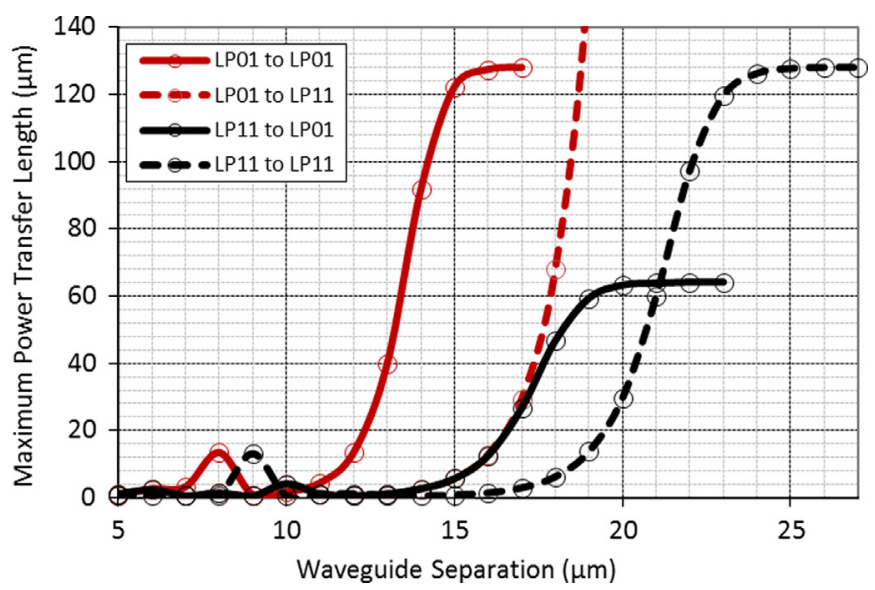

Fig. 3.5. Device lengths required to reach the calculated efficiencies with respect to waveguide separation. propagation constants of the supported modes are: $\beta_{01}=1.444714$ $490226709 \times 10^{7}$ and $\beta_{11}=1.442255268723464 \times 10^{7}$. Based on these values, phase matching condition between input second mode ( $\left.\mathrm{LP}_{11}\right)$ and output fundamental mode $\left(\mathrm{LP}_{01}\right)$ requires a grating period of $255.49 \mu \mathrm{m}$.

It should be noted that the experimental model of the coupler structure includes the input fiber and the grating-written polymer, that is, the second fiber is not mounted. This structure anticipates coupling to a continuous set of modes including radiation modes, leakage, etc. Nevertheless, the structure also includes the propagation modes of the two-fiber structure and therefore the theoretical model is valid for the analysis of the fabricated structure as well.

\subsection{Simulation results}

As presented in Section 2, the surface profiles of the grating steps have been mathematically modeled as a combination of two Gaussian functions with well-defined coefficients (see Fig. 2.1). The previously defined grating structure was implemented into simulation environment by defining the Fourier series expansion of the grating function, in order to be used in the efficiency calculations.

Using the mode fields and the theoretical model, propagation of each mode throughout the structure, as well as the coupling between each and every mode was calculated with respect to propagation distance. The results obtained for maximum achievable coupling ratios are presented with respect to waveguide separation in Fig. 3.4.

In addition, the corresponding device lengths required to reach the efficiency value calculated above are given Fig. 3.5.

\subsection{Optimization and dispersion relation}

Waveguide separation was set as the initial optimization parameter (Fig. 3.2). A minimum waveguide separation of $18 \mu \mathrm{m}$ ensures the complete cancellation of coupling from input $\mathrm{LP}_{01}$ to output $\mathrm{LP}_{01}$; whereas a maximum of $24 \mu \mathrm{m}$ prohibits complete loss of power coupled from $\mathrm{LP}_{11}$ to output modes. Favoring output $\mathrm{LP}_{11}$ over output $\mathrm{LP}_{01}$ and considering the beat-lengths of the modes coupled in-between the waveguides to allow for the modeselectivity, coupling efficiencies for the waveguide separation range $22-24 \mu \mathrm{m}$ are investigated.

Longitudinal Amplitudes Grating Period = 255.49um | d=23um

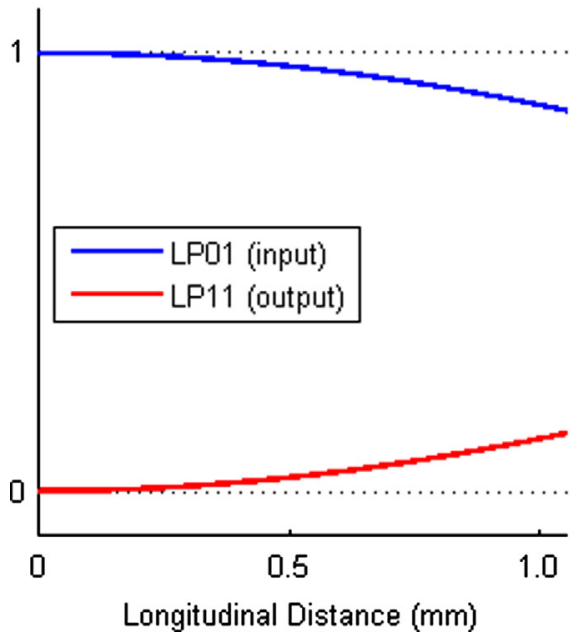

Longitudinal Amplitudes Grating Period $=255.49 \mathrm{um} \mid \mathrm{d}=23 \mathrm{um}$

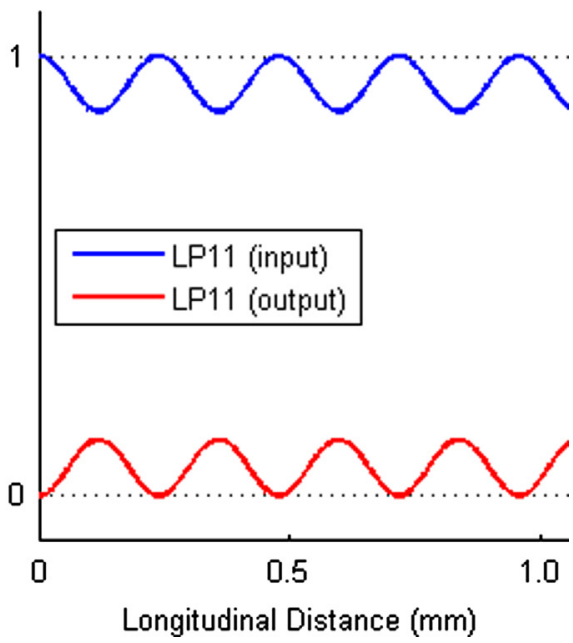

Fig. 3.6. Mode Field Propagations at Waveguide Separation $d=23 \mu \mathrm{m}$. 
Accordingly, the separation value of $24 \mu \mathrm{m}$ almost completely zeroes-out the desired mode power ( $2 \%$ coupling efficiency at $24 \mu \mathrm{m}$ ), whereas a waveguide separation of $22 \mu \mathrm{m}$ leads to a maximum power transfer ratio of $100 \%$ for $\mathrm{LP}_{01}-\mathrm{to}_{\mathrm{LP}} \mathrm{LP}_{11}$ at a transfer length of $1.932 \mathrm{~mm}$, while the coupling ratio for $\mathrm{LP}_{11}$-to$\mathrm{LP}_{11}$ is $41.94 \%$ at $97.5 \mu \mathrm{m}$, which is not realistic in terms of physical fabrication considering the grating period of $255 \mu \mathrm{m}$. On the other hand, beat-length (i.e., the longitudinal distance at which the coupled amplitude becomes zero after reaching its maximum level) at $22 \mu \mathrm{m}$ waveguide separation of input $\mathrm{LP}_{01}$ coupling is quite small and a slower transfer can be favored in order to achieve selectivity via tuning of the total device length. So, the

Table 3.1

Specifications of the final device.

\begin{tabular}{llc}
\hline $\begin{array}{l}\text { Waveguide separation } \\
\text { Device length }\end{array}$ & $\begin{array}{l}23 \mu \mathrm{m} \\
0.8354 \mathrm{~mm}\end{array}$ \\
\hline Coupling efficiency (simulation) (\%) & $\mathbf{L P}_{\mathbf{0 1}}$ to $\mathbf{L P} \mathbf{0 1}$ & 0.00 \\
& $\mathbf{L P}_{\mathbf{0 1}}$ to $\mathbf{L P}$ & 8.44 \\
& $\mathbf{L P}_{\mathbf{1 1}}$ to $\mathbf{L P}$ & 0.02 \\
& $\mathbf{L P}_{\mathbf{1 1}}$ to $\mathbf{L P}$ & 12.70 \\
\hline
\end{tabular}

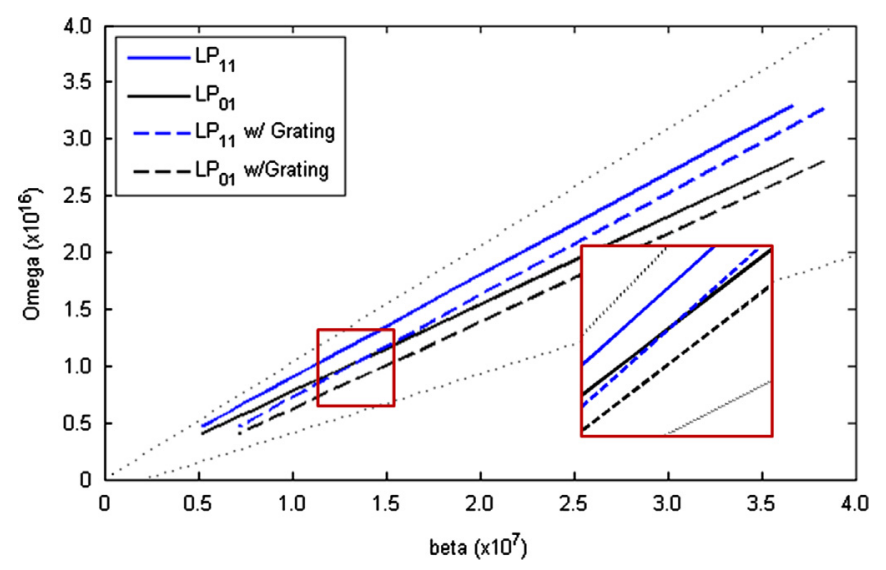

Fig. 3.7. $\omega$ vs. $\beta$ Relation for the bare fiber and fiber with grating when excited with $632.8 \mathrm{~nm}$ laser-scaled. Inset: Intersection detail.

Table 5.1

$A$ and $B$ fit coefficients before and after grating implementation.

\begin{tabular}{llll}
\hline Coefficient & $\begin{array}{l}\text { Before grating } \\
\text { implementation }\end{array}$ & $\begin{array}{l}\text { After grating } \\
\text { implementation }\end{array}$ & $\begin{array}{l}\text { Coupling } \\
\text { efficiency (\%) }\end{array}$ \\
\hline $\boldsymbol{A}$ & 1.0 & 0.96250 & 3.750 \\
$\boldsymbol{B}$ & 1.0 & 0.92365 & 7.635 \\
\hline
\end{tabular}

analysis of the mode field propagation at a waveguide separation of $23 \mu \mathrm{m}$ is performed (see Fig. 3.6).

In this case, the slow transfer rate of $\mathrm{LP}_{01}$-to- $\mathrm{LP}_{11}$ poses an advantage in terms of obtaining mode selectivity by tuning the device length. So, the device length was set as the consequent optimization parameter (Fig. 3.2).

In the simulation set discussed above, device length is set to $1.2 \mathrm{~mm}$ and the drawback is apparent in terms of $\mathrm{LP}_{11}$-to- $\mathrm{LP}_{11}$ coupling, in that the beat period coincides with the device output, causing the exact opposite of the desired outcome. A more precise study yields the optimum device length of $L=0.8354 \mathrm{~mm}$, which constitutes the final form of the designed device, presented in Table 3.1 .

Dispersion relation, namely, $\omega$ vs. $\beta$ relation of the device was investigated following the work of Sun [17] and Saleh and Teich [18]. The dispersion relations of the bare fiber and fiber with grating are presented in Fig. 3.7.

In consequence, dispersion characteristics of the $\mathrm{LP}_{01}$ mode of the native fiber intersects that of the grating induced fiber's $\mathrm{LP}_{11}$ mode at $\beta=1.4422552 \times 10^{7}$, which is also equal to the propagation constant of the $\mathrm{LP}_{11}$ mode of the native fiber. This result is in fact the targeted outcome of the analysis, confirming the final design obtained via the theoretical and simulation studies presented above Table 5.1 .

\section{Experimental realization}

The physical realization process of the device consists of the following steps: (1) Polymer synthesis-preparation of the reactant solution. (2) Polymer casting and curing. (3) Monitoring and recording the pre-grating output power. (4) Fabrication of the grating structure via photopolymerization-Laser Direct Writing. (5) Monitoring and recording the grating-induced output power. It should be noted that the complete device fabrication process is to also include casting of the second (unprocessed) polymer layer and mounting of the output fiber.

During the photopolymerization process, in addition to the direct-writing $542 \mathrm{~nm}$ He-Ne laser, a second He-Ne laser operating at $632.8 \mathrm{~nm}$ was employed to launch the fundamental mode $\left(\mathrm{LP}_{01}\right)$ and second mode $\left(\mathrm{LP}_{11}\right)$ of the fiber. Power output of the fiber was monitored using a powermeter and the output mode fields during different stages of the fabrication process was recorded using CCD camera with $720 \times 640$ pixel image sensor with $11 \times 11 \mu \mathrm{m}^{2}$ area per pixel. Schematic representation of the experimental setup is presented in Fig. 4.1.

A reflection microscope image of the fabricated grating structure on the planar side surface of the D-Fiber is presented in Fig. 4.2.

Output power levels measured during casting and grating fabrication stages are $1.057 \pm 0.15 \mu \mathrm{W}$ in bare fiber and $0.92 \pm$ $0.17 \mathrm{nW}$ for both unprocessed photopolymer coated fiber and photopolymer grating direct-written fiber. These measurements

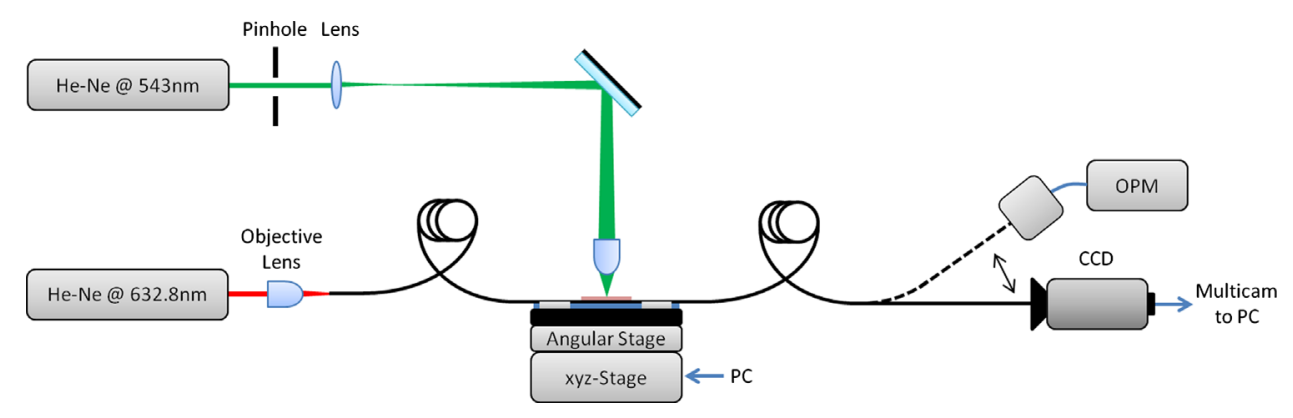

Fig. 4.1. Experimental setup. 
indicate the effect of the grating to be lower than the noise level measured at the output; therefore, the coupling efficiency was calculated via numerical analysis of the output mode fields (Fig. 4.3).

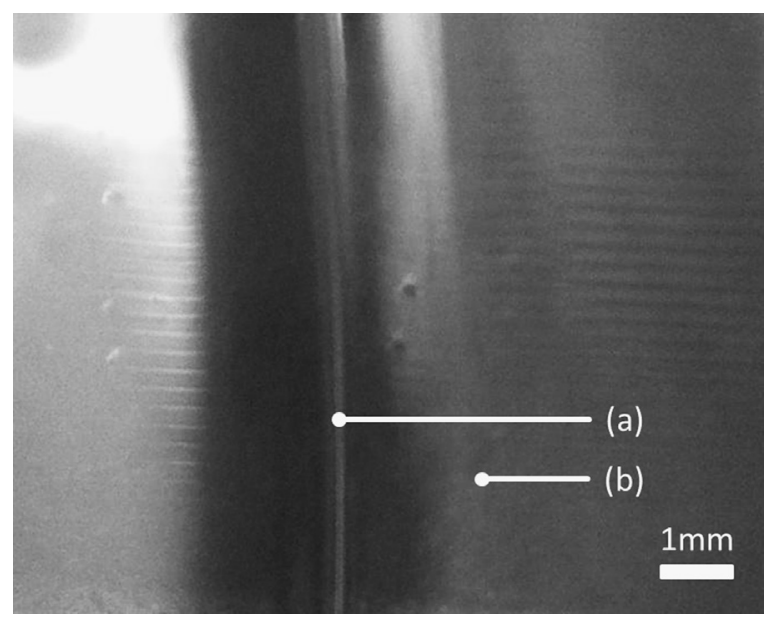

Fig. 4.2. Reflection microscopy image of the fabricated grating structure on the fiber. (a) Polymer coated grating-written fiber (b) fiber holder.

\section{Results}

Using the cross-sectional analysis of the output fiber modes, a mode-by-mode fitting was performed on the fiber outputs and power transfer between the input and output modes was calculated. Based on the mode field distributions calculated using the propagation constants earlier, the total field profiles were calculated of the form:

$F=A \cdot L P_{01}+B \cdot L P_{11}$

where $A$ and $B$ are constants used in modal fits of the fields. The measured data and the fitted curves are shown in Fig. 5.1. The noise level which can be clearly seen in the figures prevents the exact fit of the curves, while also being consistent with the noise observed in the powermeter measurements, preventing measuring the effect of the grating on the output power (see Section 4).

Accordingly, the $A$ and $B$ coefficients and corresponding coupling efficiencies are obtained as:

Results indicate a coupling efficiency of $7.635 \%$ of the $\mathrm{LP}_{11}$ mode. With these results, the actual perturbation of the light propagating inside the fiber due to the grating structure was demonstrated, which has led to almost 8\% coupling efficiency. As can be recalled, theoretical calculations showed an expected efficiency of $12 \%$, which is a bit higher than experimental results; the difference in between is associated with experimental conditions and measurement tolerances.
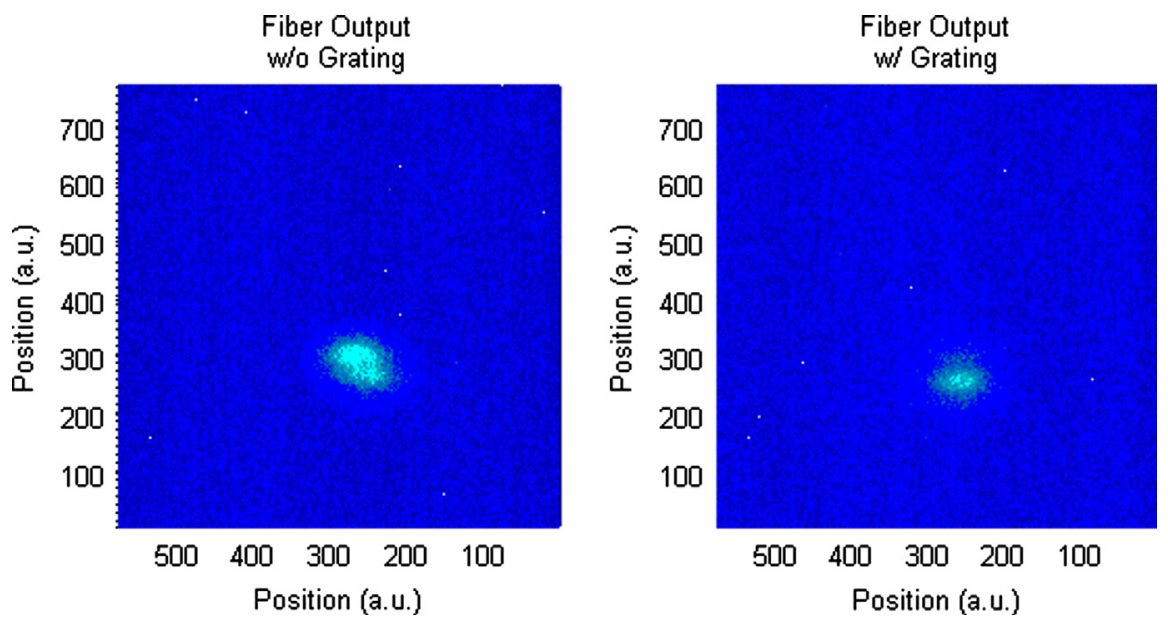

Fig. 4.3. $C C D$ fiber output field images of prior to and after grating direct writing.
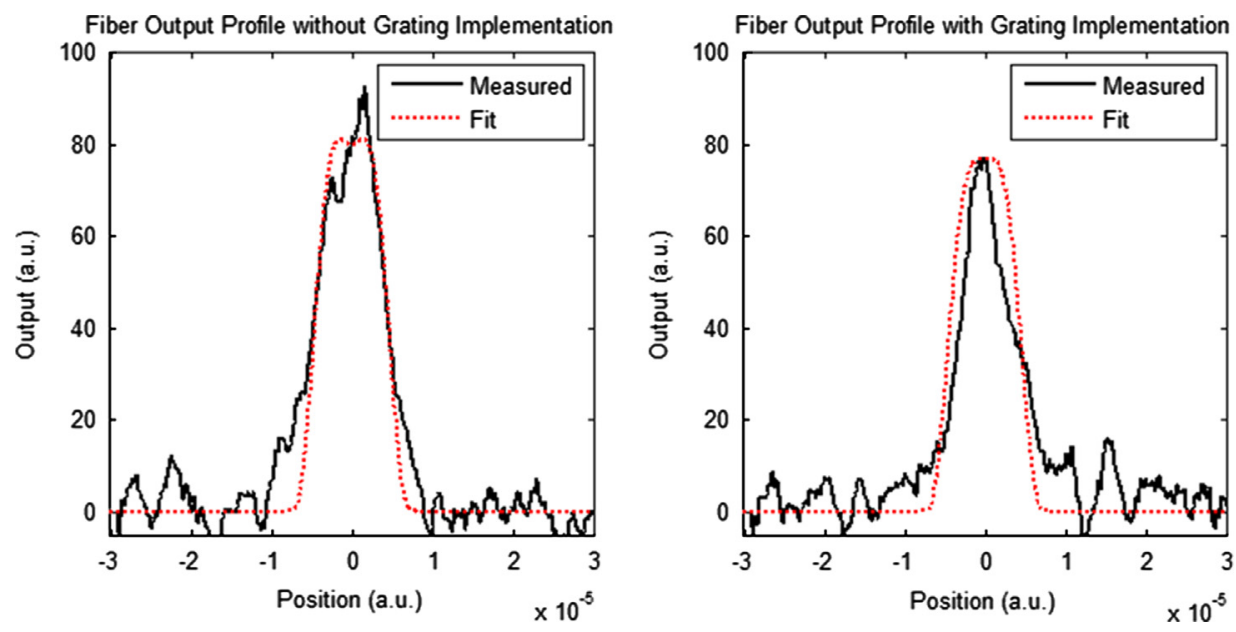

Fig. 5.1. Fiber output profiles and fits before and after grating implementation. 


\section{Conclusion}

In this study, a photopolymer grating structure capable of selectively coupling the modes of an optical fiber to an output fiber has been demonstrated in proof of principle. It has also been shown that Acrylamide/Polyvinyl Alcohol based photopolymer is an appropriate material for the fabrication of fiber integrated photonic components. The surface relief and corrugation characteristics formed as the result of the photopolymerization process have been investigated; the line shape and corrugation depths were mathematically modeled and characterized with respect to the direct-writing beam speed. In addition, the offset behavior of the line corrugations has been demonstrated.

The theoretical model of the coupler has been adapted to the device design and optimized to maximize mode-selectivity. The final device design has been implemented on the optical fiber and device functionality has been demonstrated experimentally. The resultant coupling efficiency has shown a room for improvement in the device design, in that the total coupling efficiency could be improved to further increase the mode-selectivity of the grating coupler.

Together with the simplicity of the fabrication process, the results demonstrate the proof of principle for utilizing the mechanism as the building block of various fiber-embedded optical components produced via photopolymer direct-writing, including optical switches, mode filters and add/drop multiplexers.

\section{Acknowledgment}

The authors would like to thank Dr. Mustafa M. Demir from Izmir Institute of Technology, Department of Chemistry for the assistance and experimental facilities provided during this study.
Also, the support of Dr. Emre Heves from Sabancı University Nanotechnology Research and Application Center (SUNUM) for the surface profilometer measurements is deeply appreciated.

\section{References}

[1] C. Garcia, I. Pascual, A. Costela, I. Garcia-Moreno, C. Gomez, A. Fimia, R. Sastre, Applied Optics 41 (2002) 2613.

[2] T. Sabel, S. Orlic, K. Pfeiffer, U. Ostrzinski, G. Grützner, Optical Materials Express 3 (2013) 329.

[3] A.C. Sullivan, M.W. Grabowski, R.R. McLeod, Applied Optics 46 (3) (2007) 295.

[4] W.P. Huang, H.A. Haus, Journal of Lightwave Technology 7 (6) (1989) 920.

[5] A. Yesayan, M. Piche, Optical and Quantum Electronics 36 (14) (2004) 1221.

[6] H. Yokota, M. Kobayashi, H. Mineo, N. Kagawa, H. Kanbe, Y. Sasaki, Optics Communications 281 (19) (2008) 4893.

[7] Smith, K.H. (2005). In-Fiber Optical Devices Based on D-Fiber, Ph.D. Thesis, Brigham Young University.

[8] Lowder, T.L. 2008. Surface Relief D-Fiber Bragg Gratings for Sensing Applications, Ph.D. Thesis, Brigham Young University.

[9] A. Yariv, P. Yeh, Photonics: Optical Electronics in Modern Communications, (The Oxford Series in Electrical and Computer Engineering), Oxford Univ. Press, New York, 2006.

[10] M.S. Dinleyici, C. Sümer, Optics Communications 284 (21) (2011) 50671 October 2011, ISSN 0030-4018, 10.1016/j.optcom.2011.07.038.

[11] C. Neipp, S. Gallego, M. Ortuño, A. Márquez, A. Beléndez, I. Pascual, Optics Communications 224 (1-3) (2003) 27.

[12] B. Das, J. Joseph, K. Singh, Optics Communications 282 (2) (2009) 177.

[13] Z. Liu, N. Srisanit, X. Ke, P. Wu, S. Song, J.J. Yang, M.R. Wang, Optics Communications 273 (1) (2007) 94.

[14] M. Gleeson, J. Sheridan, Journal of the Optical Society of America B: Optical Physics 26 (2009) 1736.

[15] S. Gallego, M. Ortuño, C. Neipp, A. Márquez, A. Beléndez, I. Pascual, Applied Optics 44 (29) (2005) 6205.

[16] T. Babeva, D. Mackey, I. Naydenova, S. Martin, V. Toal, Journal of Optics 12 (12) (2010) 124011.

[17] N.-H.m Sun, et al., Journal of Lightwave Technology 15 (12) (1997), Dec 1997.

[18] B.E.A. Saleh, M.C. Teich, Fundamentals of Photonics, John Wiley and Sons Inc, 2007. 